\title{
Parlamentarismusentwicklung und Politikwissenschaft
}

von Werner J. Patzelt

Dieser Beitrag geht dem Wechselverhältnis von Parlamentarismusentwicklung und politikwissenschaftlicher Parlamentarismusforschung nach. Er zeigt, welche konkreten institutionellen und politisch-kulturellen Entwicklungen die deutsche Parlamentarismusforschung bis in die neueste Zeit geprägt haben, wie sich der Parlamentarismus selbst in groben Zügen seit der Antike entwickelt hat, und wie jene institutionellen Entwicklungen sich anhand eines neueren evolutionstheoretischen Ansatzes analysieren lassen.

This paper shows how the developments of parliamentarism and of legislative research interact. It outlines which institutional and cultural developments have impacted legislative research in Germany; it sketches the history of parliamentarism since antiquity; and it presents a recent evolutionary approach to the study of developing parliamentary institutions.

\section{Das Anliegen}

Dreifachen Sinn hat die Rede vom Zusammenhang zwischen Parlamentarismusentwicklung und Politikwissenschaft. Erstens entstand mit modernen Parlamenten ein Großteil der Themen moderner Politikwissenschaft: von den Parlamentsfraktionen bis zu jenen Parlamentswahlen, in denen Parteien konkurrieren, und von neuartigen Regimetypen (etwa parlamentarischen und präsidentiellen Regierungssystemen) bis zu jener auch ein Massenpublikum erreichenden Medialisierung des Politischen, die so recht erst mit Parlamentswahlen, demokratischen Parlamenten und parlamentarischer Öffentlichkeitsarbeit aufkam. Natürlich gehen mit realer Parlamentarismusentwicklung auch weiterhin Veränderungen der politikwissenschaftlichen Agenda sowie bevorzugter Theorien einher: von der Befassung mit „,transnationalem Parlamentarismus“ und der Rolle von Parlamenten in Mehr-Ebenen-Systemen bis zum Aufstieg des delegationstheoretischen „principal-agent-Ansatzes“ zu Lasten traditioneller Lehren vom „freien Mandat". 
Zweitens gehen von der Politikwissenschaft bzw. ihren Geschwisterdisziplinen wie der Staatsrechtslehre immer wieder Impulse zur Weiterentwicklung parlamentarischer Regeln, Verfahren und Strukturen aus. Oft nimmt das die Form wissenschaftlicher Unterstützung solcher Reformprozesse an, die ohnehin von politischer Seite angestoßen werden. Beispiele sind etwa die 1969 erfolgte Kleine Parlamentsreform, die 2001 gelungene Reform des Rechts der parlamentarischen Untersuchungsausschüsse oder die 2009 unternommene Stärkung des Bundestages in der europäischen Integrationspolitik. Doch mitunter ist es auch die Staats- bzw. Politikwissenschaft selbst, von welcher Entwicklungen wenn schon nicht vorangetrieben, so doch verstetigt oder gesichert werden. Ein für die Bundesrepublik Deutschland sehr wichtiges Beispiel ist die Durchsetzung der Leitgedanken des parlamentarischen Regierungssystems anstelle früherer Präferenzen für den Weimarer Semiparlamentarismus oder für das monarchische Prinzip des Kaiserreichs.

Drittens kann die Entwicklung des Parlamentarismus selbst wichtiger Gegenstand auch einer sozialwissenschaftlich-systematischen Politikwissenschaft und nicht nur geschichtswissenschaftlich-idiographischer Forschungen sein. Einerseits mag es sich dabei um die Entwicklung einzelner Parlamente handeln, deren Entstehungs- und Umbildungsmuster sich anhand von Theorien institutionellen Lernens erfassen und erklären lassen, die den jeweils konkreten Untersuchungsfall weit übergreifen. ${ }^{1}$ Andererseits mag sich das Forschungsinteresse überhaupt auf die Entwicklungsmuster des sehr vielgestaltigen Institutionentyps des Parlaments richten, was zu Theorien der Institutionenevolution greifen lässt. ${ }^{2}$ Beide Ausprägungen einer nicht nur idiographischen, sondern gerade verallgemeinernden Analyse von Parlamentarismusentwicklung setzten in der Politikwissenschaft aber noch keine nachhaltigen Akzente. Nicht zuletzt aufgrund ihrer laufenden Chronisten- und Kommentatorenpflicht richtet die politikwissenschaftliche Parlamentarismusforschung ihren Blick lieber auf die Gegenwart sowie auf deren unmittelbare Vorgeschichte. ${ }^{3}$ Ohnehin kann eine historisch ausgerichtete Parlamentarismusforschung nur dann Nützliches für eine praktische Politikwissenschaft in Aussicht stellen, wenn Prozess- und Strukturmuster aus der Vergan-

1 Siehe etwa Demuth, C.: Der Bundestag als lernende Institution, Baden-Baden, 2009; Lempp, J.: Die Evolution des Rats der Europäischen Union, Baden-Baden, 2009.

2 Vgl. Patzelt, W.J.: Grundriss einer Morphologie der Parlamente, in: ders. (Hg.): Evolutorischer Institutionalismus, Würzburg, 2007, 483-564.

3 Als eine der hervorragenden Ausnahmen siehe Beyme, K. v.: Die parlamentarische Demokratie. Entstehung und Funktionsweise 1789-1999, 3. Aufl., Wiesbaden, 1999 (1. Aufl. 1970). 
genheit auch für Gegenwart und Zukunft wichtige Einsichten zu stiften versprechen, etwa für eine Vorab-Evaluation institutioneller Reformvorhaben. ${ }^{4}$ Gerade das wird aber mit gut eingeführten geschichtstheoretischen Argumenten vielfach bezweifelt. ${ }^{5}$ Die Folge: Geschichts- und Politikforschung fallen auseinander, Schätze institutioneller Erfahrung bleiben ungehoben - und die Parlamentarismusforschung, dem jeweils Aktuellen hinterher, löst vielerlei Bringschulden nicht ein, obschon sie sich begleichen ließen. Hierzu eine Alternative aufzuzeigen, ist - neben einem Überblick zum Wechselverhältnis von Parlamentarismusentwicklung und Parlamentarismusforschung - das Anliegen dieses Beitrags.

\section{Prägungen deutscher Parlamentarismusforschung}

Parlamente sind eingebettet in jenen - sich längst auch in Mehr-Ebenen-Systemen vollziehenden - Prozess des Regierens, bei dem vielerlei Institutionen, Netzwerke und Verhandlungssysteme zusammenwirken. Gut zeigt sich das im komplexen bundesdeutschen Zusammenwirken von - jeweils recht unterschiedlich parlamentarisch mitgestalteter - Landes-, Bundes- und Europapolitik. ${ }^{6}$ Die

4 Siehe hierzu Lempp, J.: Ein evolutionstheoretisches Modell zur Analyse institutioneller Reformen. Fallanalyse: Die Reform des Auswärtigen Amtes, in: Patzelt, W.J. (Hg.): Evolutorischer Institutionalismus, a. a. O., 599-639, und Sliwka, M.: Evolutionäres Management, in: ebd., 707-719.

$5 \mathrm{Zu}$ ihnen und zur Gegenposition siehe Patzelt, W.J.: Plädoyer für eine Rehistorisierung der Sozialwissenschaften, in: ebd., 237-283.

6 Es ist analytisch reizvoll, die Entwicklung des deutschen Landesparlamentarismus während des Aufbaus unseres 'unitarischen Bundesstaates' mit der Entwicklung des nationalen Parlamentarismus der EG- bzw. EU-Mitgliedsstaaten während des Fortgangs des europäischen Integrationsprozesses seit der Gründung der EG zu vergleichen. Ebenso erhellend ist ein Blick auf den Widerhall beider Prozesse in der Parlamentarismusforschung. Der Machtabstieg des deutschen Landesparlamentarismus wurde weitgehend nur beobachtet und beschrieben, zumal die Kompetenzausdünnung der Landtage nach Ausschöpfung der ihnen vom Grundgesetz eingeräumten legislativen Spielräume. Das - erfolglose - Ringen einzelner Landesparlamente in den 1950er Jahren um Einfluss auf das Agieren ihrer Regierungen im Bundesrat zog ohnehin kaum parlamentarismusanalytisches Interesse auf sich, und die verbleibende - wichtige parlamentarische Rolle bei der Regierungskontrolle und gesellschaftlichen Verankerung des politischen Systems geriet nicht selten auch bei Parlamentarismusforschern unter den boshaften Begriff eines 'Kreistags de luxe'. Freilich steht der deutsche Landesparlamentarismus - und nicht erst seit der zentralistischen Weimarer Republik - im Schatten der Politik auf nationaler Ebene, und haben die Landtage einen ganz anderen 'Sitz im Leben' Deutschlands als die europäischen nationalstaatlichen Parlamente im Rahmen des europäischen Integrationsprozesses. Umgekehrt hat dieser auch einen viel geringeren emotionalen und politisch-kulturellen Tiefgang als der deutsche Einigungsprozess des 19. Jh., in dem folglich die nationale Ebene viel stärker die gliedstaatlichen Parlamente in den Hintergrund drängen konnte als das der europäischen Ebene bislang mit den nationalen Parlamenten gelingt. Also besitzen diese eine stärkere Motivation, auch viel größere Chancen als einst die deutschen Landesparlamente, zur Selbstbehauptung im jeweiligen Mehr-Ebenen-System. Dieser Unterschied spiegelt sich ebenfalls in der Parlamentarismusforschung. In ihr hat nämlich das Ringen der nationalen Parlamente Europas um Mitsprache auf europäischer Ebene (für den Bundestag siehe v.a. Demuth, C.: Bundestag als lernende 
hier wichtige Eigentümlichkeit von Parlamenten besteht in ihrer Fähigkeit, auf jeder Ebene des Regierens Vernetzungen nicht-hierarchischer Art in drei Richtungen zunächst aufzubauen und sie dann, zum Zweck der Sicherung von Responsivität und Führung, effektiv zu betreiben. Nach „oben“ hin geschieht das zur jeweiligen Regierung im engeren Sinn (etwa vom Bundestag zur Bundesregierung); auf ,gleicher“ Handlungsebene hin zu Parteien und Verbänden, zu Verwaltungen und zu den Medien; und nach ,unten“ hin, zentriert um die Person des Parlamentariers, zum vorpolitischen Raum und zu einzelnen Bürgern. Seit Vertretungskörperschaften - ob als Konzilien im westgotischen Spanien oder als Ständeversammlungen seit dem späten europäischen Mittelalter $^{7}-$ zu wenigstens $^{-}$ potentiell ernstzunehmenden Machtfaktoren wurden, konnten sie jedenfalls weder von der Praxis noch von der Theorie des Regierens ignoriert, sondern allenfalls kleingehalten oder kleingeschrieben werden. Vollends in den Kernbereich praktischer, publizistischer und wissenschaftlicher Regierungslehre rückten Repräsentationsinstitutionen mit dem Aufkommen des liberalen Parlamentarismus in

Institution, a.a.O.) viel mehr Aufmerksamkeit, ja bisweilen sogar eine klar parlamentsfreundliche Parteinahme gefunden, als das während des Machtabstiegs der bundesdeutschen Landtage der Fall war. Es hatte allerdings die Parlamentarismusforschung - und nicht minder ihr Gegenstand - hier auch einen starken Verbündeten in der Verfassungsgerichtsbarkeit. Die Staatsqualität der deutschen Länder verteidigte diese traditionell eher semantisch und mehr exekutiv- als legislativfreundlich, während sie - zuletzt im Urteil des Bundesverfassungsgerichts zum Vertrag von Lissabon vom 30. Juni 2009 - die Staatsqualität Deutschlands äußerst parlamentsfreundlich mit dem Demokratieprinzip verkoppelte und so dem Bundestag mehr als nur Anlass gab, mit dem Gesetz zur "Ausweitung und Stärkung der Rechte des Bundestages und Bundesrates in Angelegenheiten der Europäischen Union" vom 22. September 2009 seine Einflussmöglichkeiten auf europäischer Ebene zumindest symbolisch klar zu stärken. Vorgearbeitet hatte der Bundestag zwar selbst schon durch seine Lernprozesse in der 15. und 16. Wahlperiode (hierzu im Detail: Demuth, C.: Bundestag als lernende Institution, a.a.O., 302-388). Doch es dürften die neuen parlamentarischen Instrumente zur Verhinderung von Ratsentscheidungen durchaus im Wege der Vorauswirkung bzw. verhaltensändernden Antizipation zu veränderten institutionellen Mechanismen in der Europapolitik führen - freilich nur solange, wie Bundestag, Bundesrat und Bundesregierung die dabei zu bewältigenden Informations- und Kommunikationslasten tragen können. Hier ist aber nach allen vergangenen Erfahrungen Skepsis abgebracht. Also kann man mit guten Gründen bezweifeln, dass bei erfolgreicher Weiterentwicklung des europäischen 'body politic' den nationalen Parlamenten Europas der Machtabstieg der Landesparlamente Deutschlands erspart bleibt. Doch zumindest wehrt sich inzwischen auch Deutschland mehr denn je dagegen, dass gliedstaatliche Parlamente auf das Verhalten ihrer Regierungen in suprastaatliche Handlungsverbünden praktisch nur ausnahmsweise Einfluss nehmen können und selbst unter den Bedingungen des Subsidiaritätsprinzips ihr Gestaltungsspielraum sich stark reduziert. Dass 'mehr Parlamentsmacht' in diesem Fall also nur 'mehr Macht für das Europäische Parlament' heißen kann, diese ihm auf Dauer aber nur zuwachsen darf, wenn - anders als heute - das Stimmengewicht der europäischen Wahlbürger quer über alle Mitgliedsstaaten der EU ziemlich gleich ist, gehört zu den noch längst nicht ausdiskutierten Themen von Parlamentarismusforschung und europäischer Integrationspolitik.

7 Hierzu und überhaupt zur Parlamentarismusgeschichte finden sich viele weiterführende Literaturangaben in Patzelt, W.J.: Morphologie der Parlamente, a. a. O., $485 \mathrm{f}$. und $521 \mathrm{f}$. 
England und Frankreich, sodann - in allen später „westlich“ genannten Demokratien - mit der Verbindung von Repräsentations- und Demokratieprinzip. In Deutschland war seit dem Vormärz der Streit um eine angemessene Rollenverteilung zwischen Parlament und Regierung sogar besonders heftig. Er gipfelte in der - weit über das Kaiserreich hinaus unser Parlamentsdenken prägenden Auseinandersetzung um den „,deutschen Konstitutionalismus“, 8 wurde nur vermeintlich geschlichtet in der Weimarer Form des angeblich „wahren“ Parlamentarismus ${ }^{9}$ und dann entschieden mit der - praktisch preußischem Beispiel folgenden - Durchsetzung des parlamentarischen Regierungssystems in ganz Deutschland: nach 1945 in den westlichen Besatzungszonen, nach 1990 in den neu gebildeten Ländern der untergegangenen DDR.

Die intellektuelle und wissenschaftliche Auseinandersetzung mit solcher Parlamentarismusentwicklung prägten in Deutschland drei Faktoren. Am folgenreichsten war, dass es hier kein organisches Werden von Repräsentationsinstitutionen gab wie in England oder in dessen zu den USA werdenden amerikanischen Kolonien. ${ }^{10}$ Parlamentarismus und Parlamentarismusforschung entwickelten sich vielmehr entlang von realen Systemumbrüchen sowie unter institutionellen Diskontinuitätsbehauptungen, welch letztere zumal die ständische Tradition des zeitgenössischen Parlamentarismus in Abrede stellten. ${ }^{11}$ Auch angesichts institutioneller Neubildungen - vom Reichstag des Norddeutschen Bundes bis zum Europäischen Parlament - entfaltete sich in Deutschland der wissenschaftliche Diskurs ums Parlament viel lebendiger als Spielart aktuellen politischen und politisch-publizistischen Argumentierens, auch als parlamentsrechtliches Schrifttum, denn als auf historische Zusammenschau setzende Analyse geschichtlich gewordener Strukturen und Funktionen. ${ }^{12}$

Zweitens wurde die geistige Spannkraft derer, die sich für den Parlamentarismus interessierten, großenteils absorbiert von jeweils politisch aufgezwungenen Grundlagendebatten. Im 19. Jh. ging es um die - oft bestrittene, seltener befür-

8 Siehe Botzenhart, M.: Deutsche Verfassungsgeschichte 1806-1949, Stuttgart, 1993.

9 Einflussreich für Deutschland: Redslob, R.: Die parlamentarische Regierung in ihrer wahren und in ihrer unechten Form, Tübingen, 1918.

$10 \mathrm{Zu}$ den letzteren siehe v.a. Squire, P.: The Evolution of American Colonial Assemblies as Legislative Organizations, in: Congress \& the Presidency, 32/2 (2005), 109-131.

11 Dagegen: Bosl, K. (Hg.): Der moderne Parlamentarismus und seine Grundlagen in der ständischen Repräsentation, Berlin, 1977.

12 Siehe hierzu exemplarisch Boldt, H.: Parlamentarismustheorie. Bemerkungen zu ihrer Geschichte in Deutschland, in: Der Staat 19/3 (1980), 385-412. 
wortete - Adaptierbarkeit des englischen oder französischen Parlamentarismus auf die vom „monarchischen Prinzip“ geprägten deutschen Verhältnisse, und zwar mit Nachwirkungen bis zur - nicht nur staatsrechtlichen - Gewaltenteilungslehre der Gegenwart. ${ }^{13}$ Seit der Weimarer Zeit musste auch immer wieder die Auseinandersetzung mit faktisch antiparlamentarischen Positionen geführt werden, die den tatsächlich entstandenen (deutschen) Parlamentarismus an Fiktionen seiner Leitideen, auch an Phantasmagorien eines erfundenen ,goldenen Zeitalters des Parlamentarismus“ maßen ${ }^{14}$ darum einen „Niedergang der Parlamente “15 diagnostizierten und deshalb nach ,zukunftsträchtigeren“ Systemalternativen verlangten. Später galt es den Abwehrkampf gegen jenen klassentheoretisch und gesellschaftspolitisch begründeten Antiparlamentarismus zu bestehen, der sich mit den kommunistischen Revolutionen im 20. Jh. verschärfte ${ }^{16}$ und seit der - nicht nur universitären - Kulturrevolution von „1968“ der deutschen Parlamentarismusforschung geradezu den Kristallisationspunkt ihrer disziplinärorganisatorischen Verselbständigung abgab. Ebenfalls in dieser Traditionslinie von Parlamentarismuskritik und „Kritik der Parlamentarismuskritik“17 stehen die Debatten der 1990er Jahre um Politik-, Parteien- und Politikerverdrossenheit sowie um einen (,weiteren“) Vertrauensverlust der Parlamente. Ihrerseits riefen sie politikwissenschaftliche Untersuchungen hervor, die - auf breiter empirischer Grundlage - die Vorwürfe u.a. mangelnder oder fehlgeleiteter Arbeitsleistung von Abgeordneten sowie der „Abgehobenheit“ von Parlamentariern überprüften. ${ }^{18}$

Drittens gab es in Deutschland während der rund 130 Jahre zwischen dem Wiener Kongress und der Neubegründung deutscher Staatlichkeit nach dem Zweiten

13 Immer noch grundlegend ist Fraenkel, E.: Ursprung und politische Bedeutung der Parlamentsverdrossenheit (1966), in: ders.: Deutschland und die westlichen Demokratien, 7. Aufl., Stuttgart u.a., 1979, 101-110, und ders.: Historische Vorbelastungen des deutschen Parlamentarismus (1959), in: ebd., 13-31; siehe auch Patzelt, W.J.: Ein latenter Verfassungskonflikt? Die Deutschen und ihr parlamentarisches Regierungssystem, in: Politische Vierteljahresschrift, 39/4 (1998), 725-757.

14 Besonders kritisch hierzu: Oberreuter, H.: Kann der Parlamentarismus überleben?, Zürich, 1977, 21-31.

15 Locus classicus ist Bryce, J.: The Decline of Legislatures, Nachdruck aus „Modern Democracies“ (2 Bde, London/New York, 1921) in: Loewenberg, G. (Hg.): Modern Parliaments: Change or Decline?, Chicago, 1971, $21 \mathrm{ff}$.

16 Als marxistisch-leninistischen O-Ton siehe Schuster, J.: Parlamentarismus in der BRD. Rolle und Funktionen des Bundestages bei der politischen Machtausübung des Imperialismus, Berlin (Ost), 1976.

17 Hierzu immer noch heranzuziehen: Wasser, H.: Parlamentarismuskritik vom Kaiserreich zur Bundesrepublik, Stuttgart-Bad Cannstatt, 1974.

18 Etwa Patzelt, W.J./Algasinger, K.: Abgehobene Abgeordnete? Die gesellschaftliche Vernetzung der deutschen Volksvertreter, in: Zeitschrift für Parlamentsfragen, 32/3 (2001), 503-527. 
Weltkrieg, also während der Herausbildungsphase des modernen deutschen Parlamentarismus, keine disziplinär integrierte, umfassende Wissenschaft von der Politik. ${ }^{19}$ Deshalb gab es, anders als seit dem späten 19. Jh. in den USA, auch keine kohärente, noch der heutigen Parlamentarismusforschung ihr Fundament legende genuin politikwissenschaftliche Befassung mit den - obendrein ihre Rolle so sehr wandelnden - parlamentarischen Institutionen. Vielmehr hatte sich die alte deutsche Kameral- und Polizeiwissenschaft in verschiedene ,politische Wissenschaften" verselbständigt, unter anderem in die politische und politisierende Geschichtsschreibung, in eine - um systematischen Gegenwartsbezug gelagerte - Doppelhistorie von politischen Ideen und politischen Institutionen, in eine ins Soziologische geweitete Wirtschaftswissenschaft, sowie ins akademische Staats- und Völkerrecht, wo die „Allgemeine Staatslehre“ sogar zu einer Art juristisch enggeführter Politikwissenschaft auf indirekter empirischer Grundlage wurde. Von diesen Wissenschaften aus, die nach ihrem herausragenden Gegenstand „Staatswissenschaften“ genannt wurden, griff - mitunter auch offen publizistisch - in die jeweils aktuelle Diskussion um den Parlamentarismus ein, wem dies ein politisches oder systematisches Anliegen war. Alle diese Praxis- und Fachbezüge sowie einzeldisziplinär-paradigmatischen Traditionsbindungen wirkten fort in der nach 1949 neu begründeten, seit den späten 1960er Jahren vom revitalisierten Marxismus in eine Krise getriebenen und ab den späten 1970er Jahren sich neu konsolidierenden deutschen Politikwissenschaft. Sie waren auch prägend für die deutsche Parlamentarismusforschung.

Diese entwickelte sich in Deutschland erst seit der Mitte der 1960er Jahre als ein auch genuin politikwissenschaftliches Forschungsgebiet. ${ }^{20}$ Seit 1969 fand sie ihr integrierendes Organ in der "Zeitschrift für Parlamentsfragen“, ihre politischparlamentarische sowie publizistische Vernetzung in der „Deutschen Vereinigung für Parlamentsfragen“. Im Zug ihrer politikwissenschaftlichen Entfaltung verdorrte der deutschen Parlamentarismusforschung allerdings mehr und mehr ihr ideen- und institutionengeschichtlicher Zweig. ${ }^{21}$ Das gilt zwar nicht für die stets hochgehaltene Reflexion auf die Erblasten des scheiternden Weimarer Par-

19 Siehe Bleek, W.: Geschichte der Politikwissenschaft in Deutschland, München, 2001.

20 Ein knapper neuerer Forschungsüberblick ist Oberreuter, H./Kranenpohl, U./Sebaldt, M. (Hg.): Der Deutsche Bundestag im Wandel. Ergebnisse neuerer Parlamentarismusforschung, 2. Aufl., Wiesbaden, 2002, 7-21.

21 Dazu kam es trotz vorbildlicher Werke wie Beyme, K.v., a.a.O., oder Kluxen, K.: Geschichte und Problematik des Parlamentarismus, Frankfurt/M., 1983, sowie inspirierender Editionen wie Rausch, $H$. (Hg.): Die geschichtlichen Grundlagen der modernen Volksvertretung. Die Entwicklung von den mittelalterlichen Korporationen zu den modernen Parlamenten, 2 Bde., Darmstadt, 1974/1980. 
lamentarismus. Doch allzu wenig Pflege fand jahrzehntelang die Schnittstelle zur ja durchaus florierenden geschichtswissenschaftlichen Analyse von Vertretungskörperschaften, etwa im Umfeld der schon 1951 gegründeten „Kommission für Geschichte des Parlamentarismus und der politischen Parteien“. Die Folgen all wurden vor einem Jahrzehnt so bilanziert: „Kaum Theorie, bloße Kumulation, dazwischen wenig echter Vergleich. ${ }^{\text {(22 } 2}$ Eben das sollte sich ändern. Hinweise auf eine zu diesem Zweck fruchtbar einzuschlagende Forschungsrichtung gibt der Blick aufs Ganze der Parlamentarismusentwicklung.

\section{Grundzüge der Parlamentarismusentwicklung}

Inzwischen haben sich Parlamente ${ }^{23}$, einer erfolgreichen Spezies ähnlich, über die ganze Erde verbreitet. Man findet sie in unzweifelhaft demokratischen Staaten wie Deutschland ebenso wie in halbautoritären Regimen vom Schlag Russlands, ja sogar in echten Diktaturen wie Simbabwe. Blickt man aber zurück in die Geschichte, so lichtet sich rasch die Reihe parlamentarischer Institutionen. Vertretungskörperschaften, die nicht föderativen Ursprungs sind und sich trotzdem schon auf den ersten Blick als Repräsentationsinstitution erkennen lassen, findet man in Asien und Afrika kaum vor dem 20. Jh., in Amerika schwerlich vor dem 17. Jh.

Bis in die Antike reicht allein die parlamentarische Tradition Europas und seiner kulturellen Vorgänger. Verstreut gibt es sogar Kunde von sehr frühen Vertre-

22 So Schüttemeyer, S.: Vergleichende Parlamentarismusforschung, in: Berg-Schlosser, D./Müller-Rommel, F. (Hg.): Vergleichende Politikwissenschaft, 3. Aufl., Opladen, 1997, 191-206, hier 195. Für die deutsche Parlamentarismusforschung ist inzwischen auf diesen Befund korrigierende Bände hinzuweisen wie Helms, L.: Politische Opposition. Theorie und Praxis in westlichen Regierungssystemen, Wiesbaden, 2006, oder Patzelt, W.J./Dreischer, S. (Hg.): Parlamente und ihre Zeit, Baden-Baden, 2009.

23 In diesem Beitrag wird grundsätzlich ein weiter Begriff des Parlaments verwendet: Jede Vertretungskörperschaft fällt unter ihn, der eine (organisations-)politische Aufgabe zumindest zugeschrieben wird. Korporativer Parlamentarismus besteht, wo immer eine Vertretungskörperschaft Gremium der Selbstregierung oder Selbstverwaltung einer Organisation ist; föderaler Parlamentarismus ist gekennzeichnet durch eine periodisch tagende Vertretungskörperschaft, die aus den realen Machtträgern oder aus Gesandten zusammenwirkender politischer Systeme besteht; beim ständischen Parlamentarismus wirken die rechtlich wie faktisch realen Machtträger eines Gebiets mit ineinander greifenden Herrschafts- oder Machtbefugnissen zusammen, wobei auch teils Territorien, teils Korporationen vertreten werden können; liberaler Parlamentarismus besteht, wenn Parlamentarier als Repräsentanten unterschiedlichster Gruppen von den Repräsentierten mit einem freiem Mandat ausgestattet sind; und demokratischer Parlamentarismus geht über den liberalen dadurch hinaus, dass es hier im Wortsinn „Abgeordnete“ gibt, die über gleiche, allgemeine, unmittelbare, geheime und freie Wahlen verlässlich an die Bürgerschaft rückgebunden sind. Zu den Vorzügen eines weiten Parlamentsbegriffs und zu den genannten Ausprägungen des Parlamentarismus siehe Patzelt, W. J.: Morphologie der Parlamente, a. a. O. 
tungskörperschaften, etwa vom Panku des Hethiterreiches im 14. Jh. v. Chr. ${ }^{24}$ So richtig fassbar werden die antiken Vertretungskörperschaften aber erst mit den nicht selten durch ein Auslosungsverfahren bestellten - boulai der griechischen poleis, nämlich ihren beratenden Gremien, ferner mit den - aus Gesandten bestehenden - Synhedrien, d.h. Bundesräten, der spätgriechischen Bundesrepubliken. Vielerlei ist auch bekannt über Vertretungskörperschaften wie den Römischen Senat oder die Kurien der römischen Provinzstädte mit ihren erblich bzw. durch cursus honorum erworbenen Sitzen, desgleichen über die - unterschiedlich bestellten und vor allem mit kultischen Funktionen betrauten - Provinziallandtage der römischen Kaiserzeit. ${ }^{25}$ Die politische Theorie der Antike interessierte sich für diese Institutionen allerdings nicht, weil sie im Rahmen des Römischen Imperiums ohnehin machtpolitisch nachrangig waren und der spätere Begriff der „Repräsentation“ noch keinen „Sitz im Leben“ hatte.

Die Brücke von der Antike über das Mittelalter zur Neuzeit schlugen dann die Synoden der alten ökumenischen Kirche, die (General-)Konzilien der römischen Kirche sowie die (General-)Kapitel der christlichen Orden. Sie alle machten die abendländische Kirche - geprägt durch römisches Recht, durch eine zunächst nicht nur faktisch, sondern auch normativ föderal-synodale Struktur, später mehr und mehr durch die ,päpstliche Monarchie“ seit dem Reformpapsttum des 11. Jahrhunderts - bis weit nach dem Streit um den „Konziliarismus“26 auf den Konzilien von Konstanz (1414-1418) und Basel (1431-1449) zu einem großen europäischen Verfassungslaboratorium. In ihm konnte der Parlamentarismus avant la lettre auch zu solchen Zeiten überdauern, da es noch kaum politische oder „staatliche“ Strukturen gab, für die er hätte nützlich sein können. Vermutlich könnte die historisch-vergleichende Parlamentarismusforschung den damaligen Diskussionen und Repräsentationspraktiken viel Erhellendes über die Entwicklung säkularer Vertretungskörperschaften sowie über deren Positionskämpfe mit einer sich mehr und mehr festigenden Zentralgewalt entnehmen. ${ }^{27}$

24 Der Panku war ein Adelsrat, in dem auch die Regenten der verschiedenen Landesteile sowie die Vasallenkönige der Hethiter vertreten waren. $\mathrm{Zu}$ seinen Rechten gehörten nicht nur die Bestätigung oder Ablehnung des vom Großkönig vorgeschlagenen Thronfolgers, sondern auch die Mitwirkung an Gesetzgebung und Vertragsabschlüssen sowie die Kontrolle über den Großkönig und seine Mitarbeiter. Siehe Brandau, B./Schickert, H.: Hethiter. Die unbekannte Weltmacht, 2. Aufl., München/Zürich, 2002, $289 \mathrm{f}$.

25 Nähere Literaturangaben hierzu und zum Nachstehenden finden sich in Patzelt, W.J.: Morphologie der Parlamente, a. a. O., $485 \mathrm{f}$. und $521 \mathrm{f}$.

26 Vgl. Tierney, B.: Foundation of the Conciliar Theory: the Contribution of the Medieval Canonists from Gratian to the Great Schism, Leiden u. a., 1998.

27 Siehe etwa Wilks, M.: The Problem of Sovereignty in the Later Middle Ages, London, 1964. 
Tatsächlich begann die fortan ungebrochene Entwicklung des abendländischen säkularen Parlamentarismus genau in den zweieinhalb Jahrhunderten zwischen der endgültigen normativen Herausbildung der päpstlichen Monarchie im 13. Jh. und deren Sieg über das ,parlamentarische Kirchenregiment“ des Konziliarismus im 15. Jh. Sie vollzog sich im Rahmen jener Ständeversammlungen (Provinzial-, Land- oder Reichsstände, mitunter auch „Generalstaaten“ genannt), in denen weltliche und geistliche Fürsten, Städte, bisweilen auch Ritter und Bauern, als „versammeltes Land“ dem Monarchen periodisch - und meist aufgrund monarchischer Einberufung - gegenübertraten. Ihre Machtgrundlage war einesteils die Wirtschafts- und Finanzkraft der Stände, andernteils ihre genau darauf gegründete Fähigkeit, in jenen Zeiten des noch fehlenden Staats- und Verwaltungsapparats für den Monarchen ,arbeitsteilige Herrschaftsdienstleistungen“ zu erbringen. Da aber jede anhaltende Verletzung von Eigentumsrechten von Vasallen und Untertanen die politische Position eines Landesherrn untergraben musste, ließen sich zusätzlich nötige Finanzmittel für einen Monarchen nur dann ohne legitimitätsgefährdende Nebenfolgen erschließen, wenn sie die Ständeversammlung auf Antrag hin bewilligte. Das aber ging selten ohne monarchische Gegenleistungen ab. Somit konnte ein rational handelnder Monarch die in einer Ständeversammlung zur Geltung gebrachten politischen Wünsche schlechterdings nicht ignorieren. Diese Ausgangslage eröffnete bald verschiedene Möglichkeiten einer institutionellen Weiterentwicklung des Verhältnisses zwischen Monarchen und Vertretungskörperschaft. Deren oft auch parallele Entfaltung macht die Parlamentsgeschichte überaus farbig, gibt der idiographischen Parlamentsgeschichtsschreibung bis heute vielerlei Stoff ab und fesselte seit je das - durchaus auch politisch-praktische - Erkenntnisinteresse jeweils zeitgenössischer Parlamentsbeobachtung und politischer Theorie.

Natürlich konnte der Monarch - wie in der Kirche der Papst - erfolgreich versuchen, sich von der Vertretungskörperschaft unabhängig zu machen. Weil für Landesherren deren wichtigster Nutzen im Bewilligen von Steuern und Angaben lag, bestand der einzuschlagende Weg darin, sich durch Landesausbau und gute Wirtschaftspolitik eigene Finanzquellen zu erschließen. Auf deren Grundlage ließ sich dann ein Verwaltungsapparat aufbauen, vielleicht gar ein stehendes Heer unterhalten. Wer aber beides besaß, konnte alsbald innenpolitischen Widerstand und zumal die Macht des hohen Adels brechen, für den die Ständeversammlungen bis dahin eine wichtige Bühne sowie ein wertvolles Machtmittel waren. Vor allem in Frankreich war die Krone darin erfolgreich und schuf den sogenannten „Absolutismus“. 
In England hingegen, wo - mit „,King in Parliament “ als Verfassungsprinzip das Gemeinwesen von Krone und Vertretungskörperschaft gemeinsam repräsentiert wurde, rief im 17. Jh. ein ähnlich auf Absolutismus abzielendes Unterfangen der Monarchie den Bürgerkrieg zwischen Krone und Parlament hervor. Er endete mit dem Sieg des Parlaments. Über die Glorreiche Revolution von 1688, über den alsbald - nämlich 1714 - zweiten parlamentarisch bewirkten Dynastiewechsel sowie über das - von 1721 bis 1742 währende - Aufeinandertreffen innenpolitisch eher desinteressierter Könige mit dem machtbewussten „Premierminister“ Sir Robert Walpole verlor die Krone dann immer mehr an Macht. Diese wuchs zunächst dem Premierminister und sodann jener Parlamentsmehrheit zu, die einst Walpole durch persönliches ,government by corruption " hinter sich gebracht hatte. Einmal als mögliche Machtbasis erkannt, konnte sich eine Parlamentsmehrheit nämlich auch aus rein politischen Überlegungen, wenn diese viele Parlamentarier strategisch miteinander verbanden, hinter einem Premierminister versammeln und ihn so der Krone gegenüber stark machen.

Diese Machtverschiebungen eröffneten einen zweiten Weg der Parlamentarismusentwicklung. Auf ihm war ab der Mitte des 19. Jahrhunderts in England ein Übergewicht des Parlaments erreicht, zu Beginn des 20. Jahrhunderts gar dessen Vormacht gegenüber der Krone. Das vollendete den Übergang zum ,parlamentarischen Regierungssystem“. Beschleunigt hatte ihn die Popularisierung und Durchsetzung des Demokratieprinzips. Wenigstens im Unterhaus wurde nämlich die Repräsentation von Ständen durch die des Volkes ersetzt, wobei - und nicht nur in England - seit dem zweiten Drittel des 19. Jahrhunderts vielerlei Wahlrechtsreformen den Kreis der Wahlberechtigten immer weiter zogen. Nach dem Ersten Weltkrieg begann er in mehr und mehr Ländern die gesamte erwachsene Bevölkerung zu umfassen. Unter Geltung des Demokratieprinzips wird sich aber einem mit dem Monopol demokratischer Wahl ausgestatteten Parlament jede anders denn demokratisch gerechtfertigte Staatsgewalt unterordnen müssen. Derzeit erleben wir Ähnliches auf europäischer Ebene: Seit es 1979 erstmals direkt gewählt wurde, erlangte das Europäische Parlament immer ausgedehntere Gesetzgebungsbefugnisse und ist dabei, die Europäische Kommission von seiner unmittelbaren Unterstützung abhängig zu machen.

Ein dritter Weg der Parlamentarismusentwicklung gabelt sich ausgangs des 18. Jahrhunderts. Damals war in England ein Machtgleichgewicht zwischen Krone und Parlament erreicht. Dieses bildeten die aus britischen Kolonien entstehenden USA in republikanischer Form als ,präsidentielles Regierungssystem“ nach. Es ließ sich obendrein als ideale Form der Gewaltenteilung zwischen Le- 
gislative und Exekutive verstehen und auch mit dem Demokratieprinzip bestens vereinbaren, sobald sowohl Kongress als auch Präsident faktisch, wenngleich nicht technisch, direkt vom Volk gewählt wurden. Gleichsam von ,natürlicher Plausibilität“, wurde das präsidentielle Regierungssystem später mehrfach ,exportiert", v.a. nach Südamerika. Sein Kennzeichen ist, dass das Bestehen der Regierung nicht vom Parlament abhängt, was dessen Mehrheit das „Mitregieren“ als zentrales Mittel der Regierungskontrolle vorenthält und die Vertretungskörperschaft auf die Rolle einer „Legislative“ beschränkt.

$\mathrm{Zu}$ dieser Rolle des Parlaments konnte es allerdings auch dadurch kommen, dass der in England weiter gehende Aufstieg der Parlamentsmacht durch ein Zusammenwirken von Krone und öffentlicher Meinung gestoppt wurde. Das geschah etwa in Deutschland und war hierzulande für das Staats- und Parlamentsdenken höchst folgenreich. Dieses kreiste um das „monarchische Prinzip“: Alle Herrschaftsgewalt wurde als zunächst einmal beim Fürsten liegend gedacht (,,vorwaltende Kronmacht"); dieser aber hatte sie mit dem Parlament hinsichtlich von Gesetzgebung und Budgetbewilligung zu teilen. Hingegen wurde ein unmittelbarer Zugriff des Parlaments auf die Staatsleitung als der „Natur der Sache“ widersprechend abgelehnt. Zwar wurde im Lauf der Zeit der „Erste Minister“ der Krone vielerorts auch von einem Mindestmaß an parlamentarischem Vertrauen abhängig, womit sich - zumal über die neu errungenen parlamentarischen Fragerechte $^{28}$ und deren Verbindung mit politischer Öffentlichkeitsarbeit - auf das Regierungshandeln weit über Budgetrecht und Gesetzgebung hinaus parlamentarischer Einfluss gewinnen ließ. Dennoch blieb gerade in Deutschland die Denkfigur recht unangefochten, dass der Regierungschef letztlich der Einsetzung durch ein mit eigenständigen Regierungsbefugnissen ausgestattetes Staatsoberhaupt bedürfe, um nicht eine ,ungebremste Parlamentsherrschaft“ an die Stelle der ,eigentlich anzustrebenden Gewaltenteilung“ zwischen Legislative und Exekutive treten zu lassen.

Wo immer dann - etwa um des Demokratieprinzips willen - ein Staat zur Volkswahl seines Oberhaupts überging, sich aber nicht auf das Gewaltenteilungsmodell des präsidentiellen Regierungssystems einlassen wollte und deshalb an der Position eines mit dem Staatsoberhaupt nicht identischen Regierungschefs fest-

28 Es ist vielsagend, dass diese alsbald ,Interpellationsrechte“ genannt wurden, denn interpellatio meint im Lateinischen eine „Störung“ oder „Unterbrechung“. Eine solche ereignet sich - so die Insinuation - im Parlament stets dann, wenn dessen ,normaler“, bislang auf Gesetzes- und Budgetberatung beschränkter Beratungsgang durch ,dazwischengeschobene“ Fragen von Parlamentariern und die folgenden Antworten von Regierungsmitgliedern ,,aufgehalten“ wird. 
hielt, entstanden Hybridsysteme. Dort wird die Regierung sowohl dem Präsidenten untergeordnet als auch vom Parlament abhängig gemacht. Das verleiht dem Parlament sowie der parlamentsgetragenen Regierung gegenüber dem Präsidenten dann Stärke, wenn die Wähler solche Parteien mit der Parlamentsmehrheit ausstatten, die Gegner des Präsidenten sind. Erlangt hingegen die Partei des Präsidenten oder eine mit ihr verbündete Partei die Mehrheit im Parlament, so sinkt die Regierung zum Arbeitsstab des Präsidenten herab. Am Fall der V. Französischen Republik gab Maurice Duverger diesem so unterschiedlich ausprägbaren Regimetyp den Namen „semipräsidentielles Regierungssystems“. Bei Betonung der - freilich höchst unterschiedliche Machtpotentiale erschlieBenden - parlamentarischen Regierungsverantwortlichkeit sollte er aber besser „semiparlamentarisches Regierungssystem“ heißen. ${ }^{29}$

Ferner konnte - so im Wesentlichen aber nur in der Schweiz seit dem späten 19. Jh. eingetreten - ein intensiv genutztes System von fakultativen Gesetzesreferenden dazu führen, dass die parlamentarische Konkurrenz zwischen den politischen Parteien weniger wichtig wurde als die Notwendigkeit, vom Volk anschließend nicht zurückgewiesene - d.h. „referendumssichere“ - Gesetze zu beschließen. Weil sich in einer solchen Lage aber keine Partei beim Referendum von ihren Konkurrenten als wählerfern vorführen lassen wollte, zog die ausgedehnte Referendumspraxis den praktizierten Regimetyp der Allparteienregierung nach sich. In ihm schrumpft die Rolle des Parlaments auf die einer personellen Kupplung zwischen der Gesellschaft und einer Regierung, die vom Parlament zwar gestellt, doch weder mit Opposition noch mit dem Risiko des Sturzes konfrontiert wird.

Im Übrigen lässt sich jenes Machtpotential, das allein schon in der institutionellen Form $^{30}$ des als demokratische Volksvertretung verstandenen Parlaments geborgen ist, auch wirkungsvoll überlagern und knebeln. ${ }^{31}$ Unter der Geltung des Demokratieprinzips geht das zwar nicht mehr so wie einst im französischen Absolutismus. Doch einesteils kann - wie beispielhaft in den realsozialistischen Staaten - der Typ der modernen Partei in sehr besonderer Weise zum tragenden

29 Vehement gegen das Konzept des Semipräsidentialismus: Steffani, W:: Semi-Präsidentialismus - ein eigenständiger Systemtyp?, in: Zeitschrift für Parlamentsfragen, $26 / 4$ (1996), 621-641.

$30 \mathrm{Zu}$ diesem Konzept sowie zu dessen Theoriekontext siehe Patzelt, W. J.: Institutionalität und Geschichtlichkeit in evolutionstheoretischer Perspektive, in: ders. (Hg.): Evolutorischer Institutionalismus, a. a. O., 287-374, sowie Lempp, J.: Evolutionäre Institutionentheorie, in: ebd., 375-413.

31 Als Fallstudie siehe Schirmer, R.: Die Volkskammer - ein ,stummes“ Parlament?, in: Patzelt, W. J./Schirmer, R. (Hg.): Die Volkskammer der DDR, Opladen, 2002, 94-180. 
Strukturelement des politischen Systems werden: Aus gleich welchen Gründen „zum Besten der Bürger“ dazu legitimiert, leitet eine - wirkungsvoll von oben nach unten geführte - Partei alle staatlichen Institutionen an, darunter auch die gesetzgebenden und regierungsbildenden Parlamente; und auf diese Weise kann sie ihren politischen Führungswillen ebenso rechtsförmlich wie willkürlich und punktgenau in den Staatswillen transformieren. Andernteils kann, wie in den „hyper-präsidentiellen“ Regimen Afrikas, durch Wahlfälschung zugunsten loyaler Parteigänger, durch Einschüchterung politischer Gegner sowie durch dies alles abrundenden Entzug parlamentarischer Infrastruktur die Macht eines Parlaments aufs praktisch Unbeachtliche zusammengestaucht werden. Zeitgeschichtliche Erfahrungen zeigen allerdings, dass die in der institutionellen Form eines Parlaments angelegten Machtpotentiale sehr rasch wieder freigesetzt werden, sobald die Einschüchterungsmacht eines Präsidenten oder einer führenden Partei zerfällt. ${ }^{32}$

Diese fünf recht unterschiedlichen Entwicklungen des Parlamentarismus sowie der von ihm Gebrauch machenden Regimetypen (parlamentarisches Regierungssystem, präsidentielles Regierungssystem bzw. konstitutionelle Monarchie, semiparlamentarisches Regierungssystem, Allparteienregierung sowie - absolutistisch, hyperpräsidentiell oder durch Parteiherrschaft - ,gefesselter“ Parlamentarismus) haben der seit dem 16. Jh. entstehenden Staatswissenschaft ${ }^{33}$ und der sich - zunächst in den in den USA - seit dem letzten Viertel des 19. Jahrhunderts disziplinär verfestigenden Politikwissenschaft ${ }^{34}$ zwar unverkennbar einen Großteil ihrer Forschungsgegenstände und politisch-praktischen Mitgestaltungsaufgaben gestellt. Sie sind aber noch nicht - und schon gar nicht einschließlich der bis in die Antike zurückreichenden Tradition von Parlamentarismus - zum ganz unumstritten verbindenden, wenn auch arbeitsteilig behandelten, Gegenstandsbereich der Parlamentarismusforschung geworden. Die eigentlich gerade so weit zu spannende Perspektive auf den Parlamentarismus und seine Entwicklung wurde nämlich folgenreich beschränkt, indem man seit dem - zunächst normativen, später auch faktischen - Durchbruch des Demokratieprinzips auf einem engen Parlamentarismusbegriff bestand: „Wirkliche“ Parlamente gäbe es nur dort, wo Abgeordnete in freien Wahlen ins Amt kämen und die so entstehende Vertre-

32 Als Fallstudie: Schirmer, R.: Machtzerfall und Restabilisierung der Volkskammer im Lauf der Friedlichen Revolution, in: Patzelt, W. J. (Hg.): Parlamente und ihre Macht, Baden-Baden, 2005, 171-215.

33 Siehe Bluntschli, J.C.: Geschichte der neueren Staatswissenschaft. Allgemeines Staatsrecht und Politik seit dem 16. Jh. bis zur Gegenwart, Aalen, 1964 (Nachdruck der 3. Aufl., München 1881).

34 Siehe Somit A./Tanenhaus, J.: The Development of American Political Science, New York, 1982. 
tungskörperschaft obendrein erheblichen politischen Einfluss besitze. Parlamente sind dann ein Institutionentyp, der keine zweihundert Jahre alt ist, und sie bestehen allenfalls ihrer formalen Hülle nach in fast allen Staaten, tatsächlich aber nur in der vergleichsweise kleinen Gruppe echter Demokratien.

Durch eine solche Verengung des Parlamentsbegriffs wird aber wissenschaftlich nicht viel gewonnen. Im Grunde erhält man nur als Abgrenzung zwischen Parlamenten und „Nichtparlamenten“, was sich viel gegenstandsnäher als Abschichtung sehr unterschiedlicher Bestellungsmodalitäten, Entwicklungsstufen und Einbettungsverhältnissen von Vertretungskörperschaften in das sie umgebende politische System verstehen lässt. Obendrein schneidet ein so enger Begriff von Parlament und Parlamentarismusentwicklung gerade jene Fragen ab, die sich doch regelrecht aufdrängen: Was haben jene vielen, geschichtlich so unterschiedlichen Formen von politischen Vertretungskörperschaften wohl gemeinsam - an Strukturelementen, an Verfahren und an Überzeugungen? Und warum haben sie gemein, was sie verbindet? Gibt es zwischen ihnen irgendwelche „Verwandtschaftsverhältnisse ${ }^{\text {، } 35}$ - etwa zwischen den englischen Houses of Parliament des 14. Jahrhunderts und dem heutigen englischen Oberhaus, zwischen dem englischen Unterhaus des 18. Jahrhunderts und dem heutigen US-Repräsentantenhaus, zwischen dem Regensburger Immerwährenden Reichstag und dem heutigen Deutschen Bundesrat, oder zwischen diesem und dem Rat der Europäischen Union? Falls ja: Wie genau entstehen solche Verwandtschaftsverhältnisse - und welche Begleiteffekte gehen mit ihnen einher? Woher kommen ferner die Unterschiede zwischen solchen Parlamenten, die zwar auf gleiche Wurzeln zurückgehen, wie durchaus Deutscher Bundestag und Volkskammer der DDR, doch die in ganz unterschiedlichen Systemumwelten agieren $?^{36}$ Was hielt überdies die einmal geprägte Form eines Parlaments selbst in recht verschiedenen Ländern auf durchaus parallelen Entwicklungswegen, etwa die Unterhäuser Kanadas und Großbritanniens, oder führte umgekehrt dazu, dass politische Vertretungskörperschaften ganz unterschiedlicher Provenienz einander heute so sehr ähneln wie inzwischen US-Senat und US-Repräsentantenhaus? Und was wäre aus alledem darüber zu lernen, welche Leistungen welche Typen von Parlamenten für jene politischen Systeme erbringen können, in denen sie agieren? Oder darüber, wel-

35 Als Skizze solcher ,parlamentarischer Verwandtschaftsverhältnisse“ siehe Patzelt, W.J.: Morphologie der Parlamente, a. a. O., 537-557.

36 Hilfreich für Analysen solcher Zusammenhänge sind die Konzepte der homologen Ähnlichkeit (ähnlich aufgrund gleichen Ursprungs), der analogen Ähnlichkeit (ähnlich aufgrund ähnlicher Umweltanforderungen) sowie der homoiologen Ähnlichkeit (ähnlich aus beiden Gründen). Siehe hierzu ebd., 511-517. 
che Reformen ein Parlament zu besseren Leistungen befähigen dürften, welche anderen es aber eher funktionsuntüchtig machten?

Antworten auf diese letzten zwei Fragen wären offenbar auch praktisch wichtig, etwa für institutionelles Hinwirken auf good governance, die erfahrungsgemäß ja nicht ohne parlamentarische Mitwirkung zu erlangen ist, oder für die Ergänzung der schon entstandenen Partialstrukturen einer global governance durch solche Vertretungskörperschaften, in denen auch die Gesellschaften - und nicht allein die Regierungen - der beteiligten Staaten institutionalisierten Einfluss besäßen. Die vorherigen Fragen versprechen immerhin historisch und regimeanalytisch interessante Antworten, die ein besseres Verständnis des Institutionentyps eines Parlaments in Aussicht stellen. Alles in allem bringt es für die Parlamentarismusforschung also nicht wirklich Vorteile, vor allem auf zeitgenössische Parlamente zu blicken und jene aus früheren Zeiten allein den Historikern zu überlassen. Was aber wäre das Besondere einer profiliert politikwissenschaftlichen Analyse der weit in die Antike zurückreichenden Parlamentarismusentwicklung, die sich durchweg in ein wirklich interdisziplinäres Verhältnis zur - im Bereich der Quellenarbeit ja nicht zu überbietenden - Geschichtswissenschaft setzte?

\section{Am Beispiel der Parlamentarismusentwicklung: „Institutionenevolution“" als Forschungsansatz ${ }^{37}$}

Die meisten Parlamente, und gut funktionierende zumal, sind keine nach Blaupausen errichteten Institutionen. Man nennt sie vielmehr ,geschichtlich gewachsen“. Doch was heißt das genau? Und was legt diese Wendung für hier und jetzt angestrebte institutionelle Parlamentsdesigns und Parlamentsreformen nahe? Gewiss informiert die reichhaltige Literatur zur Geschichte von Parlamenten sehr gut, wenngleich nicht ohne Lücken, über Geschehenes und Gewordenes, desgleichen über das Zusammenwirken teils absichtlicher, teils ganz zufälliger Ereignisse der Parlamentarismusentwicklung sowie über deren pfadabhängige Langfristfolgen. Doch was die gewonnenen empirischen Einsichten in die Prozesse des parlamentarischen Werdens und Wandels ,auf theoretischer Ebene bedeuten“, also was als den Einzelfall übergreifendes Muster, was anderes hingegen als ganz unwiederholbare Handlungskette eines Einzelprozesses gelten kann: Das bleibt noch weitgehend im Dunklen. Die historisch interessierte Parlamentaris-

37 Hintergrund dieses Abschnitts ist die im Dresdener Sonderforschungsbereich 537 „Institutionalität und Geschichtlichkeit“" entwickelte evolutionstheoretische Institutionenanalyse. Zu ihr siehe die Beiträge in Patzelt, W.J.: Evolutorischer Institutionalismus, a. a. O. 
musforschung könnte aber sehr wohl über die beschreibende Untersuchung des Gewesenen hinausgehen, ${ }^{38}$ und zwar zumal dann, wenn sie den Begriff der Evolution nicht länger nur als Gegenbegriff zu dem der Revolution oder bloß als Synonym für „Wandel“" verwendete, sondern ihn als theoretisch höchst gehaltvolles Konzept nutzte.

Das gelingt am leichtesten anhand des Begriffs der ,institutionellen Generation“. ${ }^{39}$ Er meint gerade nicht eine Art Nacheinander von „Mutterinstitution“ und „Kindinstitution“, so als ob die europäischen Landstände gleichsam die „(Groß-) Eltern" unserer Parlamente gewesen wären. Vielmehr sind parlamentarische Generationen jene mehr oder minder durch gemeinsame Sozialisationserlebnisse und Gruppenerfahrungen geprägten Alterskohorten, die zu einer gewissen Zeit in ein Parlament eintreten, dort sozialisiert werden und ihre Institution sodann - im variablen Mischungsverhältnis mit ihren Vorgängern bzw. Nachfolgern - aufrechterhalten sowie nach einiger Zeit wieder verlassen. Dergestalt verhalten sich immer neue Generationen von parlamentarischen Akteuren zu ihrer Institution wie Generationen von Lebewesen zu ihrer Art: Die letztere besteht nie ohne die sie jeweils realisierenden und ihren „Grundbauplan“ weitergebenden Individuen; doch es kommen und gehen die Mitglieder der Art oder Institution, während diese selbst unter mancherlei Wandlungen so fortdauert wie das englische Parlament seit vielen Jahrhunderten. Konkret vollzieht sich solche Generationenabfolge bei Institutionserhaltung folgendermaßen: Von jeweils aktiven Parlamentariern und Parlamentsmitarbeitern werden die tragenden handlungsleitenden Selbstverständlichkeiten ihrer parlamentarischen Strukturen in gelingenden Prozessen parlamentarischer Sozialisation an nachrückende Institutionsmitglieder weitergegeben; und werden jene ,parlamentarischen Neulinge“ nach einiger Zeit auch ihrerseits zu „wirklichkeitskonstruktiv kompetenten Mitgliedern“ ihrer Institution, so kann die weitere Tradierung parlamentarischer Selbstverständlichkeiten, Regeln und Strukturen glücken.

Allerdings kommt es immer wieder auch zu unvollkommen gelingenden Sozialisationsprozessen, gar zu scheiternden Tradierungsversuchen. Als deren Ergebnis werden sich parlamentarische Strukturen wandeln oder zurückbilden. Selten wird nämlich eine neue Parlamentariergeneration bloß eine Kopie ihrer Vorgänger sein: Es gelangen Menschen mit veränderten Hintergründen, Wissensbeständen

38 Zum Anliegen vgl. Hintze, O.: Weltgeschichtliche Bedingungen der Repräsentativverfassung (1931), in: ders.: Staat und Verfassung, 2. Aufl., Göttingen, 1962.

39 Siehe Patzelt, W.J.: Morphologie der Parlamente, a. a. O., 492-503. 
und Erfahrungen ins Parlament; institutionelle Sozialisation erfolgt unter verschiedenen, jeweils Anderes wichtig machenden Bedingungen; und nicht selten wird von Neulingen auch etliches abgelehnt, woran sich etablierte parlamentarische Akteure längst gewöhnt haben. Also können sich beim Nachrücken einer institutionellen Generation mancherlei bisher handlungsleitende Selbstverständlichkeiten verändern (allgemein: handlungsleitende „kulturelle Muster ${ }^{\text {“40 }}$ ), um die herum bislang die parlamentarischen Abläufe und Strukturen ihre Gestalt annahmen. Im Grunde kommt es beim Generationenwechsel zu einem „Mehrangebot" an neu mitgebrachten oder entwickelten Wissensbeständen, Deutungsschemata oder Handlungsbereitschaften, für deren Variationsbreite es zuvor in der Institution kaum Verwendung gab. An solcher „Überproduktion“ bzw. Variation von kulturellen Mustern setzt sodann ein zweistufiger Prozess der Selektion an.

Zunächst einmal tun die inneren (,endogenen“) Selektionsfaktoren ihr Werk. Besonders schwer oder praktisch gar nicht zu verändern sind oft solche Regeln bzw. Praxen, von denen die Plausibilität vieler weiterer Regeln oder Praxen abhängt. ${ }^{41}$ Das lässt sich leicht an der Auslegung und Anwendung der Geschäftsordnungsregeln eines Parlaments veranschaulichen. „Richtiger“ Umgang mit diesen ist an eine bestimmte, zentrale Vorstellung vom Zweck des Parlaments gekoppelt, d.h. an dessen „institutionelle Leitidee“. ${ }^{42}$ Das hat eine asymmetrische Beziehung zur Folge. Sehr wohl würde sich nämlich der Umgang mit der Geschäftsordnung dann ändern, wenn sich jene Leitidee des Parlaments wandelte, an welche die Auslegung und Handhabung der Geschäftsordnung gekoppelt ist, etwa von der Leitidee „sozialistischer Parlamentarismus“ hin zur Leitidee „Parlamentarismus im parlamentarischen Regierungssystem““ ${ }^{43}$ Hingegen würde eine der tatsächlich befolgten Leitidee des Parlaments widersprechende Veränderung von dessen Geschäftsordnung - etwa die Einführung unbe-

40 In der Sprache des hier knapp umrissenen Evolutorischen Institutionalismus wäre hier von „Memen“ oder „Memplexen“ die Rede, d.h. von jenem ,zweiten Replikator“, der auf der Ebene von Bewusstsein und Kultur in gleicher Weise wirkt wie Gene als „erster Replikator“ auf der Ebene von biochemischer Codierung und biologischer Evolution.

41 Mit derartigen „abhängigen“ Regeln bzw. Praxen ist somit die für deren Plausibilität sorgende „basale“ Regel oder Praxis gleichsam ,bebürdet“, womit ein weiterer für die Evolutionsanalyse von Institutionen zentraler Begriff eingeführt wäre; vgl. Demuth, C.: Institutionen und ihre endogenen Systembedingungen, in: Patzelt, W. J. (Hg.): Evolutorischer Institutionalismus, a. a. O., 415-448.

42 Siehe - mit weiteren Literaturhinweisen - Patzelt, W.J.: Institutionalität und Geschichtlichkeit, a.a. O., 291-300.

43 Vgl. Schirmer, R.: Machtzerfall und Restabilisierung, a.a.O. 
schränkter persönlicher Redezeit jedes Abgeordneten in den Plenardebatten des Deutschen Bundestages - für Praktiker sehr unplausibel sein, dürfte sich, falls trotzdem verwirklicht, rasch kontraproduktiv auswirken, und würde darum alsbald außer Vollzug gesetzt - oder andernfalls den Bundestag gerade bei umstrittenen Regierungsmaßnahmen machtpolitisch wirkungslos und dem Volk verächtlich machen. ${ }^{44}$

Genau in dieser hermeneutisch-praktischen Selektionsfunktion, in ihrer tragenden Rolle für weitere handlungsleitende und strukturgenerierende Selbstverständlichkeiten, besteht die „,kulturelle Bebürdung“ einer institutionellen Leitidee. Nachgeordnet gilt Gleiches für alle jene - ihrerseits von der Leitidee einer Institution getragenen - Regeln oder kulturellen Muster, von denen weitere Regeln oder kulturelle Muster ihre Plausibilität empfangen: etwa Verwaltungsvorschriften in der Hierarchie der Rechtsnormen, Tonsatzregeln im Bauplan einer barocken Fuge, vorgegebene Silbenschemata in einer poetischen Form. Weil wiederum kulturelle, soziale und institutionelle Strukturen anhand von Regeln bzw. kulturellen Mustern geschaffen und aufrechterhalten werden, lässt sich in gleicher Weise wie von „Regelbebürdungen“ oder von (kulturellen) „Musterbebürdungen “45 auch von strukturellen Bebürdungen handeln: Strukturen innerfraktioneller Mannschaftsbildung tragen - im parlamentarischen Regierungssystem - eine Regierung, stabile Regierungen supranationale Strukturen, sind also mit alledem „bebürdet“ ${ }^{46}$ Komplexere Bebürdungsstrukturen solcher Art, die obendrein über lange Zeit stabil sind, lassen sich selten anhand einer Blaupause schaffen, sondern entstehen in längeren Prozessen von Versuch und Irrtum, von Bewährung und Beibehaltung. Eben das meint die Wendung, sie seien ,geschichtlich gewachsen". Was aber da gewachsen ist, wirkt fortan als interner Selektionsfaktor: Einmal bestehende Bebürdungsstrukturen von kulturellen Mustern und sozialen Strukturen sorgen für deutlich reduzierte Realisierungs- und Tradierungschancen von solchen Veränderungen an - etwa parlamentarischen -

44 Es mag hilfreich sein, noch ein weiteres Beispiel zu geben. Ist etwa in der Leitidee eines Parlaments besonders stark die Vorstellung verankert, Parlamentarier sollten in intensiver Ausschussarbeit an Gesetzesvorlagen arbeiten, so werden all jene Veränderungsvorschläge zur Parlamentspraxis geringe Umsetzungschancen bzw. wenig Folgen haben, welche die Abgeordneten zu großer Präsenz im Plenarsaal anhalten wollen: Sie passen einfach nicht zum gelebten Leitbild, von dem aus parlamentarische Aktivitäten nach ihrer Wertigkeit beurteilt und dann auch vollzogen werden.

45 In der Sprache des Evolutorischen Institutionalismus wäre hier einfach von ,,memetischen Bebürdungen“ die Rede. Nachstehend geht der - zunächst einmal aus Anschaulichkeitsgründen eingeführte, da juristisch leicht nachzuvollziehende - Begriff der „,Regelbebürdung“ im Oberbegriff der „Musterbebürdung“ bzw. des „kulturellen Bebürdungssystems“ auf.

46 Näheres in Patzelt, W. J.: Institutionalität und Geschichtlichkeit, a. a. O., 305-314. 
Vorstellungen oder Praxen, die an jenen kulturellen Mustern und institutionellen Strukturen ansetzen, welche ihrerseits viele weitere handlungsleitende Selbstverständlichkeiten und soziale Strukturen tragen. Diese endogene Selektionswirkung äußert sich regelmäßig darin, dass man mit einer Veränderung schlechte Erfahrungen macht, woraufhin diese Neuerung an Plausibilität verliert sowie außer Gebrauch gerät. Oft auch wird man sich auf eine vorgeschlagene Veränderung gar nicht erst einlassen, weil es mit ihr bereits schlechte Erfahrungen gab oder solche für die Zukunft befürchtet werden.

$\mathrm{Zu}$ den inneren Selektionsfaktoren gehören ferner die funktionellen Bebürdungsstrukturen. Sie liegen vor, wo von der verlässlichen Erfüllung einer bestimmten Funktion auch noch die Erfüllung einer Reihe weiterer Funktionen abhängt. Falls es etwa zu den Funktionen eines Parlaments gehört, mit seiner Mehrheit eine möglichst stabile Regierung zu tragen, dann werden alle Versuche entweder fatal enden oder von vornherein verpuffen, in den Reihen der regierungstragenden Abgeordneten eine Auflockerung von Fraktionsdisziplin herbeizuführen. Meist endet derlei schon im Ansatz wegen vorauswirkender Einsicht in die wenig wünschenswerten, ja möglichst abzuwendenden Konsequenzen des Verzichts auf selbstverständliches Agieren als - scharfer Konkurrenz ausgesetzte - parlamentarische Mannschaft. Misslingt nämlich das Durchhalten von Fraktionsdisziplin, so wird im parlamentarischen Regierungssystem kein klarer Regierungskurs zu halten sein und damit auch die Parlamentsfunktion notleidend, durch Richtungskontrolle für einen solchen Kurs zu sorgen. Das wiederum werden viele Parlamentarier vorhersehen und dann, um derlei zu verhindern, selbst gegen inneres Widerstreben ihren Teil zur weiteren Aufrechterhaltung von Fraktionsdisziplin beitragen. Die hier einschlägige Funktionshierarchie endet also „oben“ in Regierungsstabilität, hat als nächstniedrigere Trägerschicht die „konstruktive Richtungskontrolle“ der Regierung, und dieser liegt wiederum „Fraktionsdisziplin“ zugrunde, welche ihrerseits in bejahtem Mannschaftsgeist gründet. In eben dieser tragenden Rolle der kulturellen Muster „Mannschaftsgeist“ und „Fraktionsdisziplin“ für die Erfüllung hiervon abhängiger Funktionen besteht ihre „funktionelle Bebürdung“".

Auch hier liegt eine asymmetrische Koppelung vor. Schwindet nämlich „Mannschaftsgeist", so wandeln sich auch die mit ihm verbundenen handlungsleitenden Selbstverständlichkeiten der „Fraktionsdisziplin“, „,konstruktiven Richtungskontrolle“ und „Regierungsstabilität“. Hingegen wird im parlamentarischen Regierungssystem sogar eine Regierung mit ganz erratischem Kurs eher sich selbst zu Fall bringen als bewirken, dass sie den parlamentarische Vorstellungskomplex 
auflöst, eigentlich solle es zwischen der Regierung und ihrer Parlamentsmehrheit solchen Mannschaftsgeist geben, der fraglose Regierungsstabilität mit gern praktizierter Fraktionsdisziplin verbindet. Im Übrigen wirken funktionelle Bebürdungssysteme als innere Selektionsfaktoren in der gleichen Weise wie kulturelle bzw. strukturelle Bebürdungssysteme: Man erlebt, typischerweise als ,ungeplante Nebenwirkung geplanter Handlungen", dass sich eine Veränderung recht nachteilig für mancherlei weiterhin als wichtig erachtete Funktionsabläufe auswirkt; und deshalb wird eine solche Änderung schnell rückgängig gemacht bzw. um ihren ursprünglichen Gebrauch gebracht - oder man lässt sich, aufgrund schon gemachter oder vorausgeahnter schlechter Erfahrungen, ohnehin nicht auf sie ein. Bei alledem zeigt sich: Wo die Träger wichtiger funktioneller oder kultureller bzw. struktureller Bürden von Veränderungen abträglich betroffen sind, werden solche Veränderungswünsche, ja selbst manche schon vereinbarten Verfahrensänderungen, in der parlamentarischen Praxis immer wieder wegselektiert werden. Im dramatischen Fall mag sich derlei so vollziehen: Ein Parlament wird durch Neuerungen in Funktionsprobleme gestürzt; es verändert sich bis hin zur Funktionsuntüchtigkeit; alsbald gerät es - wie jenes der IV. Französischen Republik - mitsamt seinem politischen System in eine Existenzkrise; und in einem neu errichteten politischen System - wie dem der V. Französischen Republik bekommt es als „rationalisierter Parlamentarismus“ seine einst die Funktionstüchtigkeit störenden Elemente einfach ,wegrationalisiert“.

Dann haben freilich auch schon die äußeren (,exogenen“) Selektionsfaktoren zu wirken begonnen. Nicht alle möglichen Regeln oder Praxen eines Parlaments sind ja in der gleichen Weise oder Wirksamkeit geeignet, das Parlament jene Leistungen erfüllen zu lassen, welcher das politische System für gutes Funktionieren bedarf oder deren Erbringung schlechterdings erforderlich ist, falls das Parlament nicht an Ansehen, Ressourcen und konkreter Macht verlieren soll. Versagt etwa in einem semiparlamentarischen Regierungssystem ein Parlament wie in der Weimarer Republik oder der IV. Französischen Republik - bei der Aufgabe, eine handlungsfähige Regierung ins Amt zu bringen oder im Amt zu halten, so verlagert sich zunächst die Macht vom Parlament weg entweder nach oben zum Staatschef oder nach unten auf die Straße. Alsbald mag ein Systemoder Regimewechsel die Folge sein - und im schlimmsten Fall dahingehend, dass das Parlament, wie der Reichstag im Nationalsozialismus oder die Staatsduma in der Sowjetunion, seine bisherige Funktion oder gar Existenz verliert. Die äußeren Selektionsfaktoren sondern also aus jenen Veränderungen, die das Ausleseverfahren innerer Selektionsbedingungen noch überstanden haben, oben- 
drein jene aus, die nicht zu den funktionellen Anforderungen der politischen Umwelt an das jeweilige Parlament passen. Darum werden nur solche Veränderungen an den bislang in Geltung befindlichen Regeln und Praxen eines Parlaments nachhaltig sein und im Wechsel parlamentarischer Generationen weiterbestehen, die sowohl in das Spektrum einer plausiblen Leitideeninterpretation passen, d.h. tragende Elemente von kulturellen und strukturellen Bürden nicht beeinträchtigen, als auch im politischen Dauerbetrieb verlässlich die Parlamentsfunktionen zu erfüllen erlauben, also weiterhin im nötigen Umfang die für den Weiterbestand des Parlaments erforderlichen Legitimitäts- und Infrastrukturressourcen erlösen lassen.

Umgekehrt wird sich eine Neuerung um so leichter durchsetzen, je weniger tief sie entweder in die Schichten handlungsleitender Selbstverständlichkeiten oder in die hierarchischen Funktionsabläufe eines Parlaments eingreift. Eben das lässt Parlamente in ihren Äußerlichkeiten und „Schnittstellendetails“ beim Zusammenwirken mit Regierungen, Parteien, Verbänden, Medien oder Einzelbürgern so verschieden werden, in ihren tragenden Elementen aber recht ähnlich bleiben. Was bei alledem an absichtsvoll oder zufällig vorgenommenen Änderungen an den kulturellen Mustern und sozialen Strukturen eines Parlaments derlei endogene und exogene Ausleseprozesse übersteht, wird jedenfalls Teil der fortan verfügbaren und wechselseitig erwarteten handlungsleitenden Selbstverständlichkeiten eines Parlaments. Anhand ihrer wird dieses dann weiterhin, und im Wechsel der es tragenden institutionellen Generationen, sozial (re-)konstruiert und kulturell repliziert. Dabei kann sich die institutionelle Form gewiss verändern: einesteils in Abhängigkeit von jenen Veränderungsraten, die beim Generationenzugang sowie bei institutionellen Sozialisationsprozessen auftreten, und andernteils in Abhängigkeit von der gemeinsamen Wirkungsweise der endogenen und exogenen Selektionsfaktoren. Eben diese Veränderungsprozesse gilt es an der Parlamentarismusentwicklung sowohl faktengetreu zu beschreiben als auch in ihrer systematischen Struktur zu analysieren. So zu verfahren überführte geschichtswissenschaftliche Studien zum Institutionenwandel in die sozialwissenschaftliche Untersuchung von institutionellen Evolutionsprozessen.

Tatsächlich trifft der Begriff der Evolution viel besser als jener des Wandels, was sich bei der Parlamentarismusentwicklung vollzieht. Das Wirken innerer und äußerer Selektionsfaktoren stiftet nämlich nachhaltige Ordnung innerhalb der tatsächlich genutzten Regeln bzw. Praxen eines Parlaments, und eben das verschafft der Entwicklung von einmal etablierten Parlamenten jene - leicht im Nachhinein, schwer aber in der jeweiligen Gegenwart zu erkennende und erklär- 
bare - Richtung weiteren Werdens, die der Begriff der „Teleonomie“ auf eine Kurzformel bringt. Das Wechselspiel von inneren und äußeren Selektionsfaktoren erlaubt nämlich nicht jede beliebige Veränderung an den kulturellen Mustern und funktionserfüllenden Strukturen eines Parlaments zu jedem beliebigen Zeitpunkt, sondern ,privilegiert“ zwei Arten von absichtsvollen oder ganz zufälligen Veränderungen: jene, die zur schon bestehenden institutionellen Form des Parlaments passen, und solche, die obendrein jenen weiterbestehenden oder sich wandelnden funktionellen Anforderungen an das Parlament gerecht werden, im Gegenzug für deren Erfüllung das Parlament weitere Ressourcenzufuhr aus seiner politischen und gesellschaftlichen Umwelt erlangt. Zwar lässt sich die Entwicklung in eine einmal gebahnte Richtung auch immer wieder versperren, desgleichen der richtungsgebende Eigensinn parlamentarischer Strukturen von außen überlagern, nämlich - wie im Fall der ,herrschenden Parteien“ des Realsozialismus - durch den machtgestützten Aufbau und Einsatz eines das alles konterkarierenden politischen Steuerungssystems. ${ }^{47}$ Doch dergleichen bedarf schon des Einsatzes gewaltiger Ressourcen oder großer Veränderungen im Gesamtgefüge eines politischen Systems. Solange beides nicht vorkommt, entfalten Parlamente somit auch selbsttragende Stabilität und Eigendynamik: Sie bewegen sich auf jenem Pfad weiter, der einst mit der Festlegung ihrer institutionellen Form, mit der Herausbildung ihrer zentralen handlungsleitenden Selbstverständlichkeiten eingeschlagen wurde.

Indem solche Evolutionsprozesse nicht nur an ganz unterschiedlichen Parlamenten, sondern überhaupt an zentralen politischen Institutionen wie Regierungen, Armeen, Verwaltungsbehörden, Parteien, Verbänden usw. in weit ausgreifenden geschichtlichen Vergleichsstudien untersucht würden, entstünde mit innovativer Theorieprägung aufs Neue eine „historische Schule“ der Staats- bzw. Sozialwissenschaften. ${ }^{48}$ In der Wirtschaftswissenschaft ist dies in Gestalt der Evolutorischen Ökonomik ${ }^{49}$ längst gelungen, die ihrerseits fruchtbar an die von der Neoklassik verdrängten historischen Schulen der Nationalökonomie anschließt. Vieles spricht dafür, dass auch der Politikwissenschaft nicht zuvörderst ihre aus der neueren Institutionenökonomik ${ }^{50}$ übernommenen Modellierungsversuche auf rational-choice-Grundlage einen besseren Beitrag zur Erhellung der Staatspraxis

47 Vgl. Patzelt, W.J.: Morphologie der Parlamente, a. a. O., 504-509, 553-555 und passim.

48 Zum Gesamtprogramm: Patzelt, W.J.: Plädoyer für Rehistorisierung der Sozialwissenschaften, a. a. O.

49 Vgl. Beinhocker, E.D.: The Origin of Wealth. Evolution, Complexity, and the Radical Remaking of Economics, London, 2007, und Dopfer, K.: The General Theory of Economic Evolution, London, 2008.

50 Vgl. Erlei, M./Leschke, M./Sauerland, D.: Neue Institutionenökonomik, Stuttgart, 2007. 
ermöglichen werden, sondern dass dies besonders gut mit einem theoretisch gehaltvollen Historischen Institutionalismus ${ }^{51}$ oder dem Evolutorischem Institutionalismus gelingt, wie er hier am Fall der Parlamentarismusentwicklung umrissen wurde.

51 Vgl. Steinmo, S./Thelen, K./Longstreth, F. (Hg.): Structuring Politics: Historical Institutionalism in Comparative Analysis, New York, 1992. 